\title{
Stroke Prevention in Atrial Fibrillation: Is Left Atrial Appendage Closure Superior to Systemic Anticoagulation?
}

\author{
Nadeev Wijesuriya* and Syed Ahsan \\ ${ }^{1}$ Deparment of Cardiology, Barts Heart Centre, St Bartholomew's Hospital, London, UK \\ *Corresponding author: Nadeev Wijesuriya, Deparment of Cardiology, Barts Heart Centre, St Bartholomew's Hospital, London, UK
}

\section{Introduction}

Atrial fibrillation (AF) is the most common arrhythmia worldwide [1], and has significant associated morbidity, including increased risk of stroke. Stroke prevention in non-valvular AF (NVAF) is a dynamic, rapidly evolving and challenging field. Oral anticoagulation (OAC) is well established as the gold standard in stroke prevention for patients meeting risk criteria defined by the CHA2DS2-VASc score [2]. However, these patients are often elderly, with multiple co-morbidities including ischaemic heart disease (IHD), chronic kidney disease and frailty [3] which increase risk of bleeding. Clinicians face daily conundrums on how to balance these risks with the benefits of stroke protection. Left Atrial Appendage closure (LAAC) is an emerging technology which some believe may help to resolve these issues.

\section{Oral Anticoagulation in Stroke Prevention}

The benefit of Vitamin $\mathrm{K}$ antagonists (VKA) such as warfarin for stroke prevention in NVAF was documented over 2 decades ago by the SPAF (Stroke Prevention in Atrial Fibrillation) trials [4]. A more recent Cochrane meta-analysis [5] demonstrated that VKA conferred a $64 \%$ benefit over no treatment, and a $37 \%$ benefit over aspirin monotherapy. The major advancement in the last decade has been the advent of Direct Oral Anticoagulants (DOACs). Dabigatran, Rivaroxaban, Apixaban and Edoxaban. The DOACs subverted the risks of sub-/supra-therapeutic treatment and multiple pharmacological interactions that were inherent to VKA, thus reducing the need for regular monitoring. The DOACs outperformed VKA in large randomised control trials, all 4 demonstrating non-inferiority in stroke prevention, with reduced risks of major bleeding [6-9]. As such, the consensus guidelines have expressed a preference for DOACs over VKA in stroke prevention for NVAF patients, with a $1 \mathrm{~A}$ level of recommendation [2].

\section{Challenges of Oral Anticoagulation}

Despite its' advantages over VKA, there are several drawbacks associated with DOAC therapy. Risk of haemorrhage remains a significant limitation. All agents displaying major bleeding exceeding $3 \%$ per year, with particularly increased risks of gastrointestinal bleeding for rivaroxaban and dabigatran [9]. Treatment of patients with IHD also remains problematic due to the need for concomitant antiplatelet therapy. PIONEER-AF PCI [10] compared rivaroxaban plus a P2Y [11] inhibitor versus warfarin plus dual antiplatelet therapy (DAPT) for patients with NVAF undergoing PCI (percutaneous coronary intervention) with stenting. Both arms displayed similar efficacy, and rivaroxaban was associated with a significantly lower risk of clinically significant bleeding $(16.8 \%$ vs $26.7 \%, \mathrm{p}<0.001)$. Similar results have been demonstrated for both dabigatran [11] and apixaban [12]. This improved safety profile is reflected in the the most recent consensus guidelines, where DOAC are advocated in preference to VKA in patients undergoing PCI [13]. However, it is clear that clinically significant bleeding rates are still prohibitively high $(10-15 \%)$ for all DOAC agents when added to an antiplatelet, compared to the bleeding rates for DAPT, which is roughly $2 \%$ [14]. OAC compliance is also a major issue. $25-55 \%$ of patients are reported to be non-compliant with chronic cardiovascular medications [15]. The reasons for this include patient-related factors (e.g. socio-economic barriers), medication-related factors (e.g., cost, side effects) and providerrelated factors (e.g., a lack of follow-up). Paradoxically, compared to VKA, lack of regular monitoring for DOACs limits the physicians' ability to ensure compliance. This can be highly detrimental given their short half-lives, where discontinuation opens a larger window of risk to the patient. This window of risk is also disadvantage of any OAC strategy with regards to situations such as mandatory discontinuation for surgical procedures [16]. Given these inherent challenged, it may be that an alternative strategy may be superior to systemic anticoagulation, especially in certain circumstances.

\section{Left Atrial Appendage Closure as a Stroke Prevention Strategy}

The Left Atrial Appendage (LAA) has long been implicated in AF-related stroke. It's anatomy and blood flow characteristics 
predispose to blood stasis and thrombus formation in AF [17], and echocardiographic studies have demonstrated that LAA thrombus is responsible for over $90 \%$ of AF-related strokes [18]. Historically, open surgery was the only option for closure of the LAA; however, in more recent years, newer technologies have emerged, including endocardial closure devices (Watchman, Amplatzer), pericardialapproach epicardial ligation (LARIAT), and open/thoracoscopic epicardial clipping (Atriclip). Surgical LAAC has been performed concomitantly with surgical AF ablation, valve surgery and coronary artery bypass grafting (CABG) for decades. However, data is limited to observational cohorts and small randomized studies. A retrospective review of patients undergoing mitral valve surgery suggested a reduction in stroke risk for those who had LAAC [19] (3.4\% vs $17 \%, \mathrm{p}=0.01$ ). The LAAOS I [20] and II [21] pilot studies demonstrated the safety of concomitant LAAC plus CABG, and suggested a reduction in stroke rate in the LAAC arm. However, neither was adequately powered for stroke outcomes. The LAAOS III randomized trial is currently underway, investigating the additional benefit of LAAC to OAC therapy in patients with $\mathrm{AF}$ undergoing CABG [22].

Thus far, 2 randomized control trials have compared VKA to LAAC with the Watchman Device. PROTECT-AF [23] demonstrated non-inferiority of LAAC compared with VKA therapy, but with a higher rate of peri-procedural complications. The subsequent PREVAIL [24] trial had vastly improved safety outcomes, likely driven by increased operator experience, but failed to meet noninferiority with regards to 12-month efficacy. However, the 5-year outcomes from these 2 trials demonstrated that LAAC provided stroke protection comparable to VKA, with reductions in major bleeding and mortality [25]. A separate analysis revealed that LAAC had a statistically significant net clinical benefit over VKA of $1.42 \%$ events per year [26]. In the first year after device implantation, there was a non-significant benefit for VKA because of peri-procedural complications of LAAC. However, the balance shifted between 1 to 2 years follow-up in favour of LAAC. No studies have compared LAAC with DOAC therapy: this is the aim of the ongoing PRAGUE-17 study [27]. As yet, there is no randomized data examining epicardial devices such as LARIAT or Atriclip, although both have US FDA approval for LAAC based on observational data. A large US registry demonstrated good safety outcomes for LARIAT, with a $2.2 \%$ acute complication rate, and 95\% acute procedural success rate [28]. Pillarisetti et al compared retrospective outcomes of patients treated either with LARIAT or Watchman, showing no difference in thromboembolism outcomes [29]. Registry data from patients receiving the Atriclip and discontinuing NOAC revealed a relative risk reduction of $87.5 \%$ in ischaemic stroke rate, compared to what would have been expected in a group of patients with similar CHA2DS2-VASc scores [30].

\section{Perspective: Current and Future Directions for LAA Closure}

Whilst LAAC shows promise as a strategy for stroke prevention in NVAF, no data thus far suggests superiority efficacy over OAC. Concerns about peri-procedural complications and cost mean current indications are limited to patients with contraindications to systemic anticoagulation [31], generally due to high bleeding risk. LAAC also may be used concomitantly with cardiac surgery, where the additional risk is minimal. Serious thought should be given to performing LAAC in every AF patient undergoing CABG, especially considering the high bleeding risks of combination OAC and antiplatelet therapy. Potential use may expand if LAAC is used adjunctively with other procedures as part of a rhythm control strategy. The LAA is implicated in recurrence of persistent AF following catheter ablation [32], and both the LARIAT and Atriclip devices have been shown to provide electrical as well as mechanical isolation [33], which could be effective in reducing AF recurrence. The aMAZE trial will examine the anti-arrhythmic effects of LARIAT when combined with catheter ablation [34], and if successful, LAAC may deliver a crucial " 2 birds with 1 stone" quality of life impact on a complex and difficult to treat group of patients.

\section{References}

1. Lloyd Jones DM (2014) Cardiovascular health and protection against CVD: More than the sum of the parts? Circulation 130(19): 1671-1673.

2. Kirchhof Paulus, Benussi S, Kotecha D, Ahlsson A, Atar D, et al. (2016) 2016 ESC Guidelines for the management of atrial fibrillation developed in collaboration with EACTS. Eur J Cardiothoracic surg 50(5): e1-e88.

3. Gage BF, Waterman AD, Shannon W (2001) Validation of Clinical Classification Schemes Results From the National Registry of Atrial Fibrillation. JAMA 285(22): 2864-2870.

4. (1994) Warfarin versus aspirin for prevention of thromboembolism in atrial fibrillation: Stroke Prevention in Atrial Fibrillation II Study. Lancet 343(8899): 687-691.

5. Hart Robert G, Pearce LA, Aguilar MI (2007) Meta-analysis: antithrombotic therapy to prevent stroke in patients who have nonvalvular atrial fibrillation. Ann intern medicine 146(12): 857-867.

6. Connolly Stuart J, Ezekowitz MD, Yusuf S, Eikelboom J, Oldgren J, et al. (2009) Dabigatran versus warfarin in patients with atrial fibrillation. $\mathrm{N}$ Engl J Med 361(12): 1139-1115.

7. Giugliano Robert P (2013) Edoxaban versus warfarin in patients with atrial fibrillation. New England Journal of Medicine 369(22): 2093-2104.

8. Granger CB, Alexander JH, McMurray JJ, Lopes RD, Hylek EM, et al. (2011) Apixaban versus warfarin in patients with atrial fibrillation. N Engl J Med 365(11): 981-992.

9. Patel MR, Mahaffey KW, Garg J, Pan G, Singer DE, et al. (2011) Rivaroxaban versus warfarin in nonvalvular atrial fibrillation. N Engl J Med 36(10): 883-891.

10. Gibson CM, Mehran R, Bode C, Halperin J, Verheugt FW, et al. (2016) Prevention of bleeding in patients with atrial fibrillation undergoing PCI. N Engl J Med 375(25): 2423-2434.

11.Cannon, Christopher P (2017) Dual antithrombotic therapy with dabigatran after PCI in atrial fibrillation. N Engl J Med 377(16): 15131524.

12. Lopes RD, Heizer G, Aronson R, Vora AN, Massaro T, et al. (2019) Antithrombotic therapy after acute coronary syndrome or PCI in atrial fibrillation. New N Engl J Med 380(16): 1509-1524.

13. Lip Gregory YH (2018) Management of antithrombotic therapy in AF patients presenting with ACS and/or undergoing PCI: A Summary of the Joint Consensus Document of the European Heart Rhythm Association (EHRA), European Society of Cardiology Working Group on Thrombosis, European Association of Percutaneous Cardiovascular Interventions (EAPCI) and European Association of Acute Cardiac Care (ACCA) endorsed by the Heart Rhythm Society (HRS), Asia-Pacific Heart Rhythm Society (APHRS), Latin America Heart Rhythm Society (LAHRS): 28472850 . 
14. Garg Pallav (2015) Balancing the risks of bleeding and stent thrombosis: A decision analytic model to compare durations of dual antiplatelet therapy after drug-eluting stents. American heart journal 169(2): 222233.

15. Ho PM, Bryson CL, Rumsfeld JS (2009) Medication adherence: its importance in cardiovascular outcomes. Circulation 119(23): 30283035.

16. Spyropoulos AC, Douketis JD (2012) How I treat anticoagulated patients undergoing an elective procedure or surgery. Blood 120(15): 29542962.

17. Markl M, Lee DC, Furiasse N, Carr M, Foucar C, et al. (2016) Left atrial and left atrial appendage 4D blood flow dynamics in atrial fibrillation Circ Cardiovasc Imaging 9(9): e004984.

18. Blackshear JL, Odell JA (1996) Appendage obliteration to reduce stroke in cardiac surgical patients with atrial fibrillation. Ann Thorac Surg 61(2): 755-759.

19. García-Fernández MA, Pérez-David E, Quiles J, Peralta J, García-Rojas I, et al. (2003) Role of left atrial appendage obliteration in stroke reduction in patients with mitral valve prosthesis: A transesophageal echocardiographic study. J Am Coll Cardiol 42(7): 1253-1258.

20. Healey JS1, Crystal E, Lamy A, Teoh K, Semelhago L, et al. (2005) Left Atrial Appendage Occlusion Study (LAAOS): results of a randomized controlled pilot study of left atrial appendage occlusion during coronary bypass surgery in patients at risk for stroke. Am Heart J 150(2): 288293.

21. Whitlock RP, Vincent J, Blackall MH, Hirsh J, Fremes S, et al. (2013) Left atrial appendage occlusion study II (LAAOS II). Can J Cardiol 29(11): 1443-1447.

22. Whitlock R, Healey J, Vincent J, Brady K, Teoh K, et al. (2013) Rationale and design of the Left Atrial Appendage Occlusion Study (LAAOS) III. Ann Cardiothorac Surg 3(1): 45-54.

23. Holmes DR, Reddy VY, Turi ZG, Doshi SK, Sievert H, et al. (2009) Percutaneous closure of the left atrial appendage versus warfarin therapy for prevention of stroke in patients with atrial fibrillation: A randomised non-inferiority trial. Lancet 374(9689): 534-542.

24. Holmes DR Jr, Kar S, Price MJ, Whisenant B, Sievert H, et al. (2014) Prospective randomized evaluation of the Watchman Left Atrial Appendage Closure device in patients with atrial fibrillation versus longterm warfarin therapy: The PREVAIL trial. J Am Coll Cardiol 64(1): 1-12
25. Reddy VY, Doshi SK, Kar S, Gibson DN, Price MJ, et al. (2017) 5-year outcomes after left atrial appendage closure: From the PREVAIL and PROTECT AF trials. J Am Coll Cardiol 70(24): 2964-2975.

26. Brouwer Tom F, et al. (2019) Net Clinical Benefit of Left Atrial Appendage Closure Versus Warfarin in Patients With Atrial Fibrillation: A Pooled Analysis of the Randomized PROTECT-AF and PREVAIL Studies. Journal of the American Heart Association 8(23): e013525.

27. Osmancik Pavel (2017) Interventional left atrial appendage closure vs novel anticoagulation agents in patients with atrial fibrillation indicated for long-term anticoagulation (PRAGUE-17 study). American heart journal 183: 108-114.

28. Lakkireddy Dhanunjaya, Muhammad R Afzal, Randall J Lee, Hosakote Nagaraj, David Tschopp, et al. (2016) Short and long-term outcomes of percutaneous left atrial appendage suture ligation: results from a US multicenter evaluation. Heart rhythm 13(5): 1030-1036.

29. Pillarisetti Jayasree, Yeruva Madhu Reddy, Sampath Gunda, Vijay Swarup, Randall Lee, et al. (2015) Endocardial (Watchman) vs epicardial (Lariat) left atrial appendage exclusion devices: Understanding the differences in the location and type of leaks and their clinical implications. Heart Rhythm 12(7): 1501-1507.

30. Caliskan Etem (2018) Epicardial left atrial appendage AtriClip occlusion reduces the incidence of stroke in patients with atrial fibrillation undergoing cardiac surgery. Ep Europace 20(7): e105-e114.

31. Michael Glikson, Rafael Wolff, Gerhard Hindricks, John Mandrola, A John Camm, et al. (2020) EHRA/EAPCI expert consensus statement on catheter-based left atrial appendage occlusion-an update. EP Europace 22(2): 184-184.

32. Di Biase Luigi (2016) Left atrial appendage isolation in patients with longstanding persistent AF undergoing catheter ablation: BELIEF trial. Journal of the American College of Cardiology 68(18): 1929-1940.

33. Nishimura Marin, Florentino Lupercio-Lopez, Jonathan C Hsu (2019) Left atrial appendage electrical isolation as a target in atrial fibrillation. JACC Clinical Electrophysiology 5(4): 407-416.

34. Lee Randall J (2015) Percutaneous alternative to the Maze procedure for the treatment of persistent or long-standing persistent atrial fibrillation (aMAZE trial): Rationale and design. American heart journal 170(6): 1184-1194.

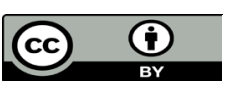

This work is licensed under Creative Commons Attribution 4.0 License

To Submit Your Article Click Here: Submit Article

DOI: $10.32474 /$ LOJPCR.2020.02.000127

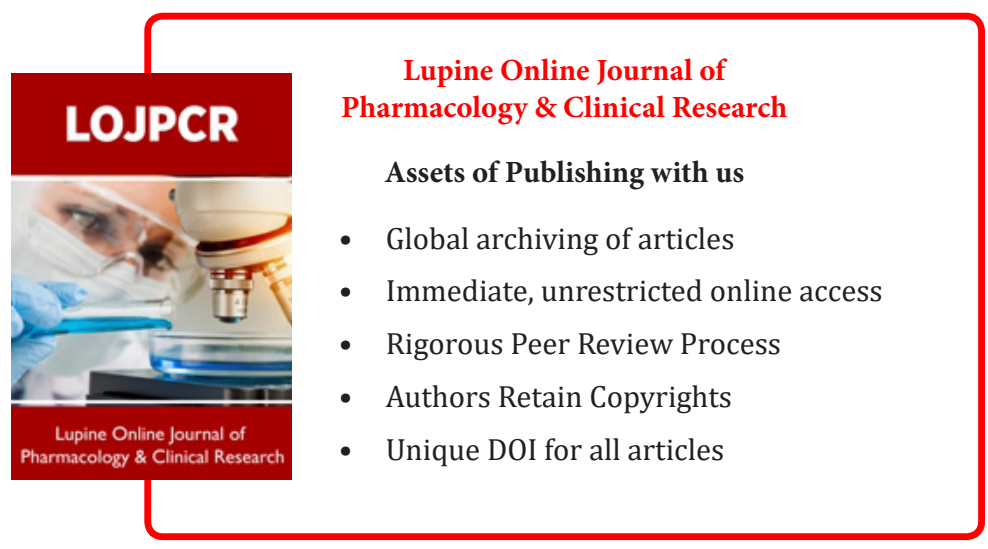

